\title{
Análisis de las huellas de manufactura del material malacológico de Tumbes, Perú
}

Analyse des traces de manufacture du matériel malacologique de Tumbes, Pérou Analysis of the traces of manufacture of the malacological material of Tumbes, Peru

Adrián Velázquez Castro, Emiliano Melgar Tísoc y Anne Marie Hocquenghem

\section{CpenEdition}

Journals

Edición electrónica

URL: http://journals.openedition.org/bifea/4730

DOI: $10.4000 /$ bifea.4730

ISSN: 2076-5827

Editor

Institut Français d'Études Andines

Edición impresa

Fecha de publicación: 1 abril 2006

Paginación: 21-35

ISSN: 0303-7495

\section{Referencia electrónica}

Adrián Velázquez Castro, Emiliano Melgar Tísoc y Anne Marie Hocquenghem, «Análisis de las huellas de manufactura del material malacológico de Tumbes, Perú », Bulletin de l'Institut français d'études andines [En línea], 35 (1) | 2006, Publicado el 08 abril 2006, consultado el 01 diciembre 2020. URL : http://journals.openedition.org/bifea/4730 ; DOl : https://doi.org/10.4000/bifea.4730

\section{$\Theta \oplus \Theta \Theta$}

Les contenus du Bulletin de l'Institut français d'études andines sont mis à disposition selon les termes de la licence Creative Commons Attribution - Pas d'Utilisation Commerciale - Pas de Modification 4.0 International. 


\title{
Análisis de las huellas de manufactura del material malacológico de Tumbes, Perú
}

\author{
Adrián Velázquez Castro* \\ Emiliano Melgar Tísoc ${ }^{* *}$ \\ Anne Marie Hocquenghem***
}

\begin{abstract}
Resumen
Con la finalidad de corroborar las bien fundadas sospechas de que fueron instrumentos de piedra los que habían servido para desgastar y cortar el material malacológico trabajado en los sitios arqueológicos tumbesinos, unos fragmentos provenientes del taller de Rica Playa fueron analizados mediante microscopía electrónica de barrido (100X-1 000X). La comparación entre las huellas de desgastes y cortes observadas en los restos prehispánicos y las que resultaron del uso de una lasca de piedra pizarra con filos cortantes, recogida en asociación con estos artefactos en el taller de Rica Playa, en conchas y caracolas actuales de las mismas especies, confirma que fueron estos instrumentos líticos los que se utilizaron para trabajar el material malacológico en tiempos prehispánicos. Demuestra además la validez de los métodos de análisis desarrollados en el marco del Proyecto «Técnicas de manufactura de los objetos de concha del México Prehispánico» del Museo del Templo Mayor del Instituto Nacional de Antropología e Historia.
\end{abstract}

Palabras clave - microscopía electrónica de barrido, material malacológico, huellas de manufactura, talleres prehispánicos, Rica Playa, Tumbes, Perú

\section{Analyse des traces de manufacture du matériel malacologique de Tumbes, Pérou}

\section{Résumé}

Pour vérifier l'impression bien fondée que c'étaient des instruments lithiques qui avaient servi à frotter et couper le matériel malacologique travaillé sur les sites archéologiques de Tumbes, quelques fragments provenant de l'atelier de Rica Playa ont été analysés par microscopie

* Museo del Templo Mayor, Instituto Nacional de Antropología e Historia. E-mail: adrianveca@yahoo.com

** Museo del Templo Mayor, Instituto Nacional de Antropología e Historia. E-mail: melgare@hotmail.com

${ }^{* * *}$ Instituto Francés de Estudios Andinos, IFEA. E-mail: amhocque@ec-red.com 
électronique de balayage (100X-1 000X). La comparaison entre les traces de frottage et de découpe observées sur les restes préhispaniques et celles produites par l'utilisation d'un fragment de schiste aux bords tranchants, trouvé en association avec les artefacts provenant de l'atelier de Rica Playa, sur des coquilles et conques actuelles des mêmes espèces, confirme que ce sont bien ces instruments lithiques qui ont été utilisés pour travailler le matériel archéologique préhispanique. Ce qui démontre aussi la validité des méthodes d'analyses développées dans le cadre du projet «Técnicas de manufactura de los objetos de concha del México Prehispánico» du Musée du Templo Mayor de l'Institut National d'Anthropologie et d'Histoire.

Mots clés - microscopie électronique de balayage, matériel malacologique, traces de manufacture, ateliers préhispaniques, Rica Playa, Tumbes, Pérou

\title{
Analysis of the traces of manufacture of the malacological material of Tumbes, Peru
}

\begin{abstract}
To confirm the hypothesis that objects of mollusk shell from archaeological sites in Tumbes, Peru, had been worked using stone tools, some fragments of pieces from the Rica Playa's PreHispanic workshop were analyzed by experimental archaeology. Modern shells of the same species as the Rica Playa assemblage were abraded and cut using an archaeological stone tool; the wear patterns produced were compared with the archaeological pieces using Scanning Electron Microscopy (100x-1 000x). Apart from the knowledge of the specific tools used to work shell objects in the Andean Region, this paper confirms the validity of the methodology proposed by the Project «Técnicas de Manufactura de los objetos de concha del México Prehispánico», at the Templo Mayor Museum, Mexico City.
\end{abstract}

Key words - scanning electron microscopy, malacological material, traces of manufacture, PreHispanic workshops, Rica Playa, Tumbes, Peru

\section{INTRODUCCIÓN}

El estudio de la tecnología es de suma importancia para el conocimiento de las sociedades, pues ésta se refiere al conjunto de elementos sociales y materiales con los que el hombre modifica su entorno. Dentro de este ámbito se incluyen las herramientas y los productos que con ellas se elaboran, como conocimientos, comportamientos, actitudes y significados, los cuales son compartidos por grupos y personas — eventualmente la sociedad en su conjunto-, siendo transmitidos de una generación a otra (Lemonnier, 1986: 154; Pffaffenberger, 1988: 241; Schiffer, 1992: 44).

Dentro de la tecnología pueden reconocerse procesos técnicos — también llamados cadenas de operación-, que son la serie de pasos que se siguen desde que el material está intacto hasta que el objeto se encuentra totalmente terminado (Leroi-Gourhan, 1943; 1945; Lemonnier, 1986: 149 y Schiffer, 1992: 51). En cada una de las fases de estas secuencias de actividades, los productores deben tomar decisiones frente a un espectro variable de posibilidades, restringidas por factores 
ambientales, históricos, sociales y culturales (Lemonnier, 1986: 153; Schiffer, 1992: 51). No existen limitantes externos a los grupos humanos lo suficientemente poderosos para ser los únicos causales de todas las tomas de decisiones de las cadenas de operación (Gosselain, 1992:580); es por ello que se ha planteado que la tecnología es una construcción social en que se ven reflejados desde principios de organización hasta aspectos ideológicos; ello incluso ha llevado a proponer que puede ser un importante indicador de etnicidad, constituyendo lo que es llamado «estilo tecnológico» (Stark, 1999: 27).

Usualmente, en la arqueología las evidencias que se utilizan para llevar a cabo los estudios tecnológicos, son los llamados indicadores directos de la producción: herramientas y desechos de manufactura, así como piezas en proceso descartadas por defectos de algún tipo. Desafortunadamente no siempre es posible plantear con seguridad que un implemento fue empleado para la elaboración de un determinado objeto por la mera asociación en el depósito arqueológico, ya que con frecuencia estos indicios provienen de contextos secundarios - como basureros - o removidos. De la misma manera, aquellos elementos que proceden de contextos votivos - como ofrendas - carecen de los mencionados indicadores directos de la producción, ya que aparecen totalmente terminados.

¿Cómo estudiar entonces los objetos que se encuentran en este último caso? ¿Cómo se puede comprobar la utilización de una determinada herramienta en un proceso productivo? En el presente artículo se presentará una propuesta a este respecto a través del análisis de algunos de los materiales malacológicos procedentes del sitio prehispánico de Rica Playa (Tumbes, Perú), los cuales aparentemente formaron parte de un taller de manufactura especializado; aún cuando en asociación con estos elementos aparecieron lo que parecen ser las herramientas empleadas para la elaboración de los objetos de concha, la remoción de los contextos arqueológicos por obras modernas crea incertidumbres al respecto. Para solucionar este problema se ha realizado arqueología experimental y se han analizado las huellas de manufactura, tanto arqueológicas como experimentales, tomando como base la metodología empleada por el proyecto «Técnicas de manufactura de los objetos de concha del México Prehispánico».

\section{EL PROYECTO «TÉCNICAS DE MANUFACTURA DE LOS OBJETOS DE CONCHA DEL MÉXICO PREHISPÁNICO»}

La arqueología experimental parte del supuesto de que una herramienta particular, empleada de una manera específica, debe producir rasgos característicos y diferenciables entre sí. Desde el año de 1997, se han desarrollado en el Museo del Templo Mayor dos proyectos sobre materiales arqueológicos de concha con esta orientación (Velázquez, 1999; 2004; Velázquez et al., 2004). Es importante aclarar que los exoesqueletos calcáreos de los moluscos fueron de los materiales más apreciados por las culturas del México precolombino, sirviendo para la manufactura de objetos ornamentales y votivos; por lo cual en la mayoría de los casos se carece de los indicadores directos de la producción. Hasta el momento, se han llevado a cabo 467 experimentos, realizando las modificaciones empleadas para fabricar las piezas, con el utillaje y los procesos que por diferentes fuentes de información (contextos arqueológicos, documentos históricos y propuestas previas de otros investigadores) pueden suponerse que fueron usadas en el México precortesiano. El análisis y caracterización de las huellas de manufactura de los ejemplares experimentales, y su comparación con los rasgos presentes en los materiales arqueológicos, es el punto nodal de la investigación, ya que a través de ello es posible descartar algunas de las técnicas probadas y proponer otras como las más probables. Ello se lleva a cabo en tres niveles de observación: macroscópicamente (simple vista), con microscopía estereoscópica de bajas amplificaciones (10X-63X), y mediante microscopía electrónica de barrido (100X1000X); si bien cada nivel proporciona información útil, es a través del último que pueden obtenerse resultados más precisos, siendo posible distinguir el empleo de materiales específicos, cuyas huellas son indiferenciables con las otras dos técnicas. 
Hasta el momento se han revisado materiales de colecciones procedentes de diferentes áreas culturales (zona maya, centro de México y centro de Veracruz), habiéndose logrado identificar formas particulares de elaboración de objetos de concha propias de sitios y épocas determinadas. Quizás en un futuro sea posible poder llegar a definir estilos tecnológicos.

\section{EL MATERIAL MALACOLÓGICO}

\section{1. Antecedentes}

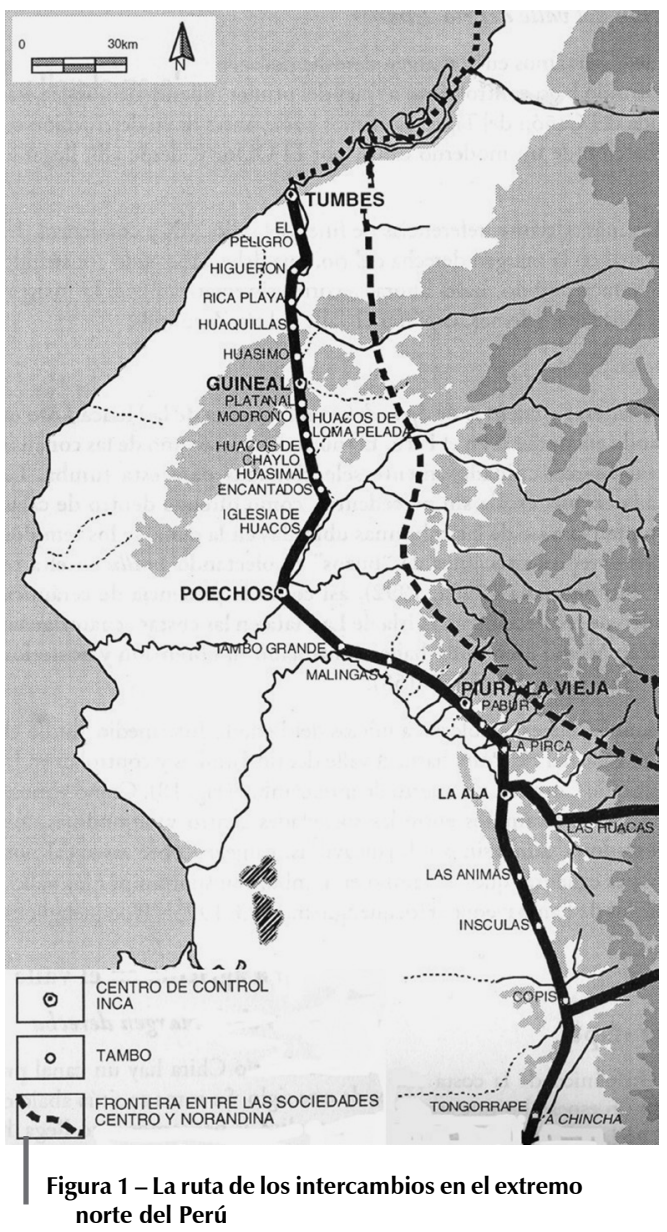

Hasta la desembocadura del río Tumbes llegaba, a partir de 900 d.C., la vía marítima seguida por las canoas y balsas que navegaban a lo largo del litoral del Pacífico, entre el golfo de California y el de Guayaquil, transportando hombres y diversos productos con alto valor de intercambio. Para estas embarcaciones la navegación se volvía más difícil hacia el sur, en contra del viento austral y de la corriente de Humboldt (Hocquenghem, 1993; 1998: 128-134,160-172; 1999). Los productos norteños se desembarcaban en el puerto prehispánico de Tumbes y se transportaban hacía el sur por la vía terrestre que controlaban primero los sicanes desde los valles de Lambayeque, luego los chimus desde los de Trujillo y finalmente, los incas desde el Cusco. Este camino se utilizó durante todo el Periodo Intermedio Tardío y el Horizonte Tardío y fue seguido, en 1532, por Pizarro y sus huestes en ruta hacia Cajamarca (Hocquenghem, 1994; 1998: 219 256). Venía por la margen izquierda del río, desde el océano hasta el actual municipio de Corrales, donde se ubican las ruinas del centro administrativo y ceremonial de Tumbes, descrito por los cronistas, hoy llamado Cabeza de Vaca. De allí pasaba por los tambos El Peligro e Higuerón y llegaba al de Rica Playa para seguir hacia el sur por los cerros de Amotape (fig.1).

Diversos productos de intercambio circulaban a lo largo de esta ruta, pero en el marco de este estudio nos interesan las conchas de moluscos (pelecípodos y gasterópodos) recolectadas en las aguas calientes al norte del golfo de Guayaquil, que llegaban por vía marítima hasta Tumbes y que se trabajaban en los talleres del extremo norte peruano. Se ubicaron y estudiaron dos de estos talleres, uno en el centro administrativo y ceremonial de Tumbes, Cabeza de Vaca, y otro en el tambo de Rica Playa (Hocquenghem, 1993; Hocquenghem \& Peña Ruiz, 1994).

Federico Kauffmann Doig (1987) atrajo la atención sobre el taller de material malacológico de Tumbes, Cabeza de Vaca. Años más tarde Manuel Peña Ruiz (1993, ms) observó restos de este taller, conchas y caracolas de los géneros Spondylus y Strombus y otras especies, notando la presencia de especímenes no modificados, centenares de desechos, pedazos en diferentes etapas de trabajo y piezas terminadas. Asociadas a estos materiales, encontró varias herramientas líticas, cantos rodados, piedras porosas y lascas de pizarra con filos cortantes. 
En enero de 1993, Anne Marie Hocquenghem y Manuel Peña Ruiz (1994) visitaron las ruinas del tambo de Rica Playa, del cual Georg Petersen había publicado un plano en 1958 (Pertersen, 1962: fig. 2). En la parte baja del sitio ubicaron los cimientos de los muros prehispánicos tal como estaban indicados en este plano. Además en la parte alta, viniendo de Tumbes y entrando en Rica Playa, a la derecha del camino, observaron los restos de una estructura, casi completamente destruida que no figuraba en el plano de Petersen y que parecía haber sido un taller de material malacológico. Poco antes de esta visita una máquina había vuelto a abrir el camino destruido por las fuertes lluvias que cayeron a raíz del Fenómeno El Niño de 1992 y, al ensancharlo, habían quedado expuestos en el talud algunos restos de conchas y caracolas así como de herramientas de piedra, en particular lascas de pizarra con filos cortantes. Además al aplanar el terreno encima del camino, para facilitar

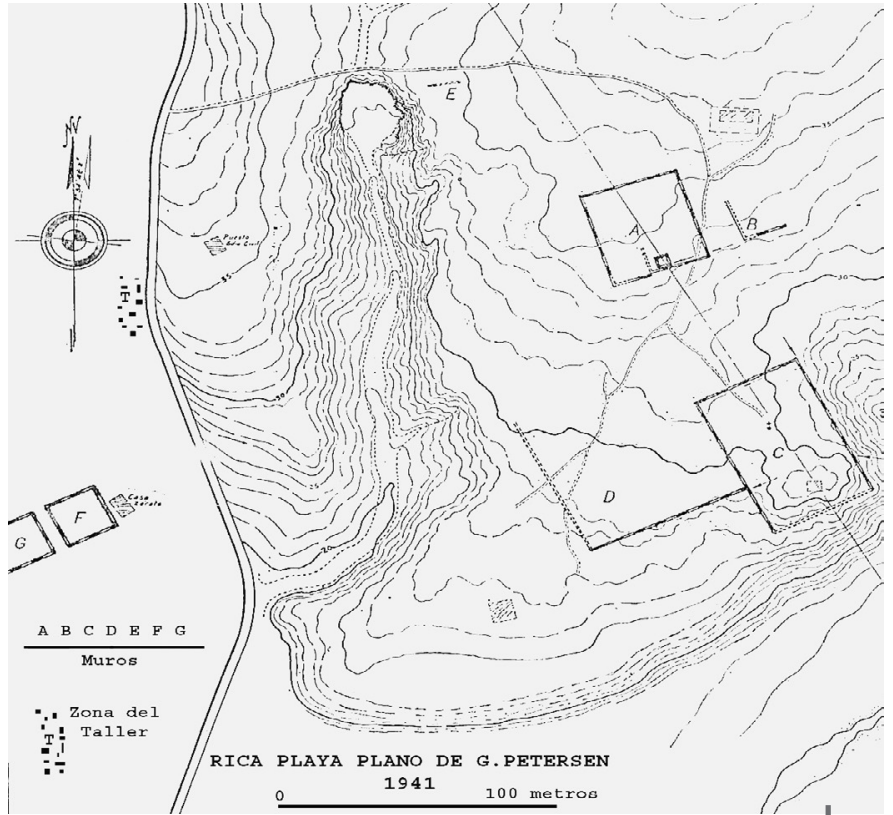

Figura 2 - Plano del tambo de Rica Paya y ubicación del taller de material malacológico

la construcción de una casa, la maquina había dejado visibles en la superficie más evidencias de talla de material malacológico. Los lugareños donaron unos ejemplares de conchas y caracolas talladas así como de lascas de pizarra con filos cortantes que tenían huellas de uso que habían recogido en este sitio. Estos materiales eran similares a los encontrados en Cabeza de Vaca, lo que indicaba que se debían haberse utilizado las mismas técnicas de manufactura en los dos talleres tumbesinos.

En base a la asociación de material malacológico tallado y de instrumentos líticos en los sitios de los talleres de Cabeza de Vaca y de Rica Playa, así como a la observación de los cortes en V en los restos de moluscos, se supuso que este material se trabajaba con herramientas de piedra y se tallaba con filudas lascas de pizarra. Apoyaban esta hipótesis los resultados de las experiencias del artesano tumbesino Félix Morán Davies que, con herramientas similares, lograba tallar conchas y caracolas de las mismas especies que las recogidas en los talleres prehispánicos, obteniendo cortes similares en V (Hocquenghem \& Peña Ruiz; 1994).

Es de notar que en 2003, cuando Anne Marie Hocquenghem volvió a Rica Playa, pudo constatar que se había construido todo un pueblo encima de los cimientos del antiguo tambo. El camino había sido una vez más destruido por las fuertes lluvias que habían caído a raíz del Fenómeno El Niño de 1997-1998 y de nuevo reconstruido y no quedaban huellas visibles del taller de material malacológico.

En un libro póstumo de Carlos Zevallos Menéndez (1995: 47-90), así como en una recopilación de los trabajos de Olaf Holm, editada por Karen Stothert (2001: 130-150), se encontraron algunos datos sobre la talla del material malacológico en la península de Santa Elena en el sur del Ecuador. De San Pablo, un sitio Valdivia ocupado entre 3000 y 1600 a.C. y excavado en 1959 por Zevallos y Holm, provienen cucharas de concha asociadas con herramientas líticas, «escariadores» y «limas-cuchillos» — también llamadas «sierras»—, quizás de areniscas, que son de formas similares a las de los instrumentos provenientes de los talleres tumbesinos. Estos artefactos evidencian la antigüedad de la tecnología de la talla de concha y caracolas en el sur 
ecuatoriano y deja pensar que en el extremo norte peruano siguió siendo utilizada hasta 1532. En 2004, con el fin de corroborar las bien fundadas sospechas de que las lascas de pizarra con filos cortantes habían servido para desgastar y cortar el material malacológico, unos fragmentos del material removido en Rica Playa fueron entregados a los investigadores Adrián Velázquez Castro y Emiliano Melgar Tísoc, del proyecto «Técnicas de manufactura de los objetos de concha del México Prehispánico» con sede en el Museo del Templo Mayor, en la ciudad de México.

\section{2. La colección analizada}

Los once fragmentos de material malacológico estudiados presentan claros indicios de trabajo; casi en su totalidad corresponden a bivalvos, de las especies Spondylus princeps (siete), Anadara grandis (dos) y Pinctada mazatlanica (uno); solamente uno de ellos es de un univalvo, Strombus galeatus (fig. 3a, b, c, d, e)

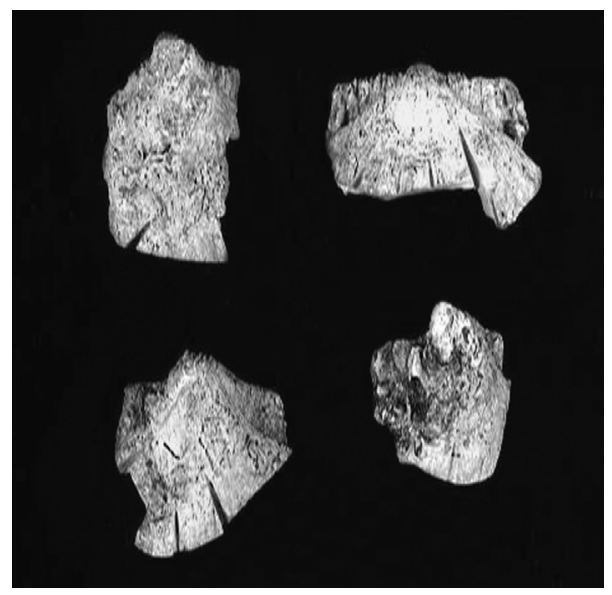

a

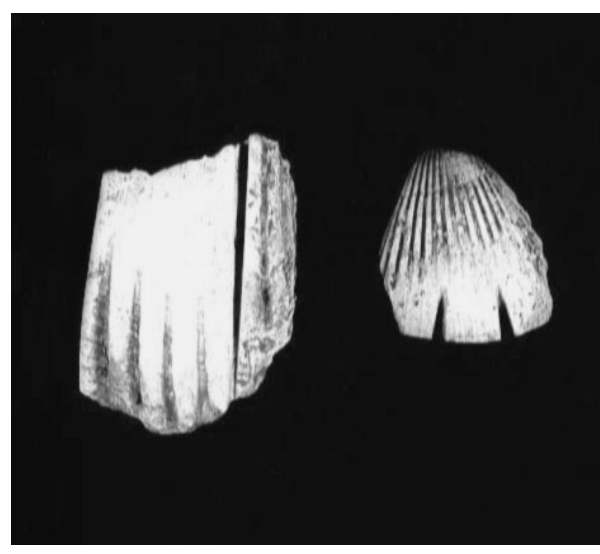

C

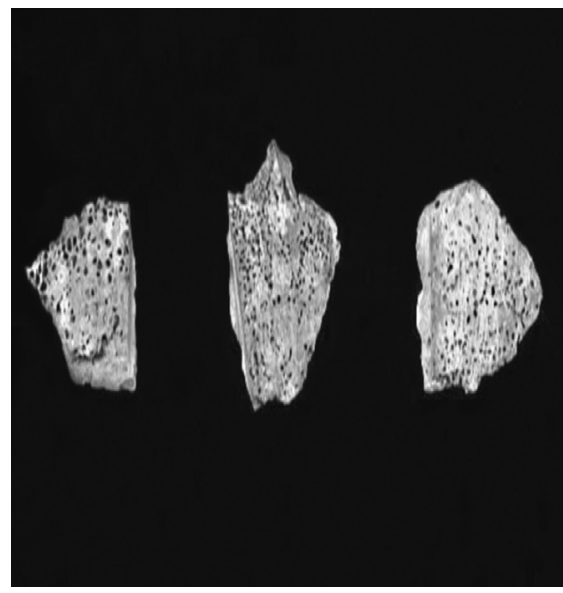

b

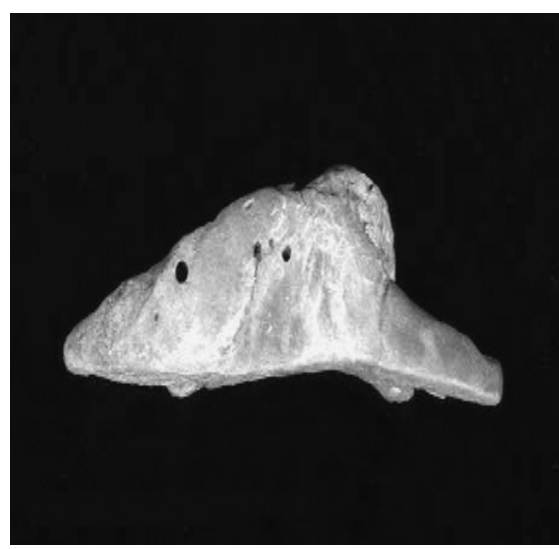

d 


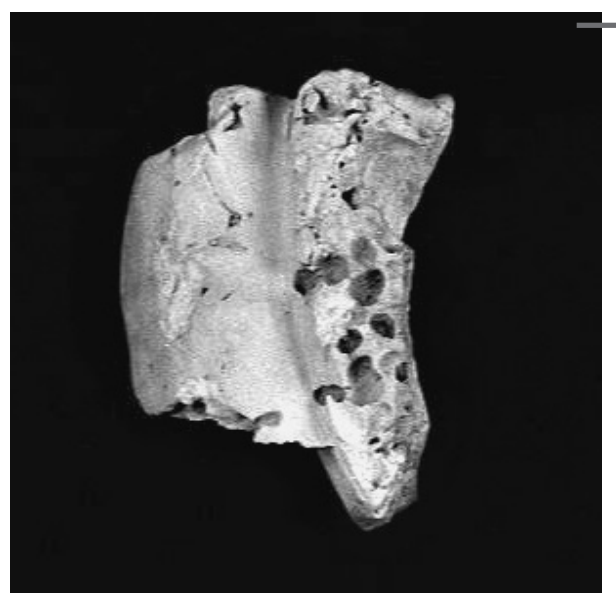

Figura 3 - Vistas de algunas de las piezas arqueológicas recuperadas en Rica Playa:

a) fragmentos de Spondylus princeps correspondientes a las charnelas; b) fragmentos de Spondylus princeps correspondientes a los márgenes ventrales y partes medias de las valvas;

c) fragmentos de Anadara grandis; d) fragmento de Pinctada mazatlanica, y

e) corte de la espira de Strombus galeatus

Todos los ejemplares evidencian procesos de desgaste superficial para eliminar total o parcialmente la ornamentación natural de los especímenes biológicos (costillas y espinas). Es conveniente aclarar que como desgaste se entiende el proceso de trabajo consistente en la abrasión del material con un agente de mayor dureza y rugosidad, en el cual pueden o no intervenir abrasivos como la arena. Dicho proceso sirve para eliminar determinadas partes de la concha, corregir cortes e incluso dar forma a los objetos, dejando en la mayoría de los casos rayones apreciables a simple vista. Por su parte, el pulido es una técnica de acabado mediante la cual se trata de difuminar las huellas del desgaste, generando una superficie lisa y homogénea; éste se aplica mediante fricción con abrasivos de grano fino. También presentan huellas de corte que en varios casos presentan un reborde irregular, resultado de una ruptura por presión o percusión. En términos generales podría pensarse que se trata de residuos de trabajo, de partes quizás no aprovechables de las valvas, en los que quedan evidencias del corte de otros fragmentos. Cuatro trozos de Spondylus princeps, el de Pinctada mazatlanica y uno de Anadara grandis corresponden a las zonas de articulación de las valvas, siendo apreciables en la mayor parte de ellos los dientes cardinales y fosetas; llama la atención que en el ejemplar de A. grandis únicamente se halle el umbo, estando ausente el resto de su charnela característica, la cual quizás fue removida por percusión, por los rebordes irregulares que se observan en la zona. Dos trozos de $S$. princeps y uno de $A$. grandis fueron obtenidos de partes cercanas al margen ventral; un fragmento de la primera especie corresponde a la parte central de la valva y el de Strombus galeatus se obtuvo de la espira, ya que se aprecia una de las suturas. 


\section{3. Experimentos y análisis de la colección}

Los experimentos que se llevaron a cabo consistieron en cortes y desgastes superficiales en ejemplares actuales de las mismas especies de la colección arqueológica, empleando en todos los casos una herramienta de pizarra hallada en el mismo asentamiento (fig. 4); ésta consiste en una lasca aplanada de forma irregular, con filos cortantes en varios de sus lados, que al inicio de los experimentos medía $11,25 \mathrm{~cm}$ de largo, $6 \mathrm{~cm}$ de ancho y $0,6 \mathrm{~cm}$ de alto.

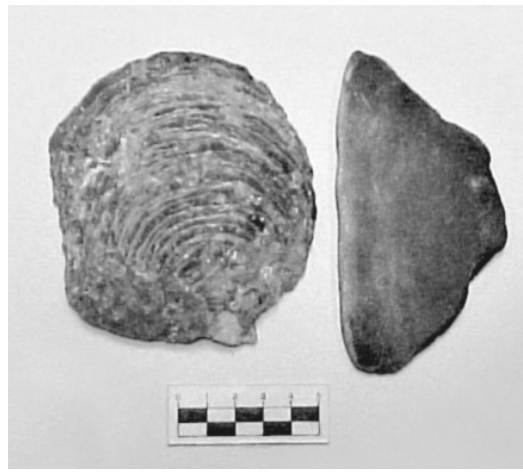

a

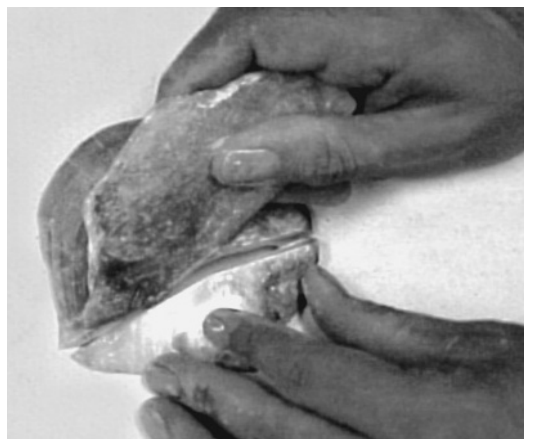

C

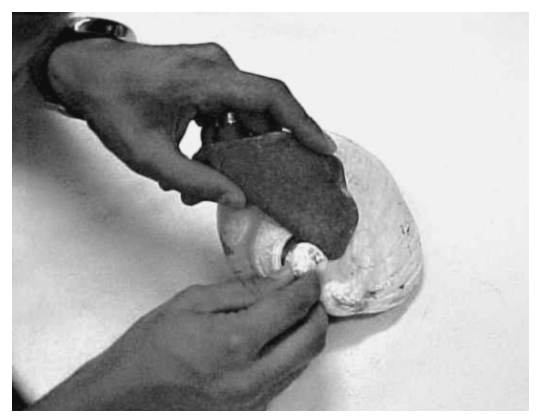

e

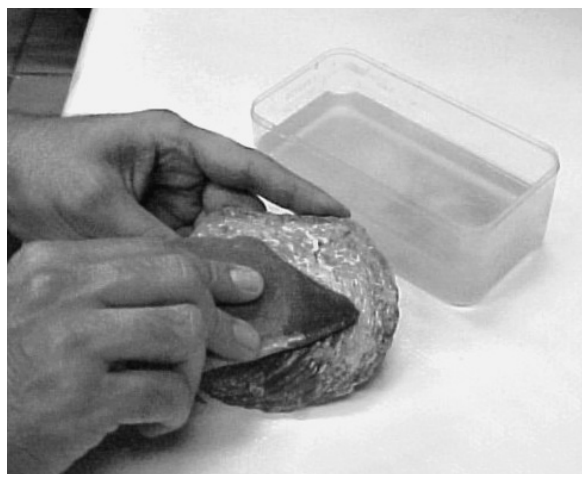

b

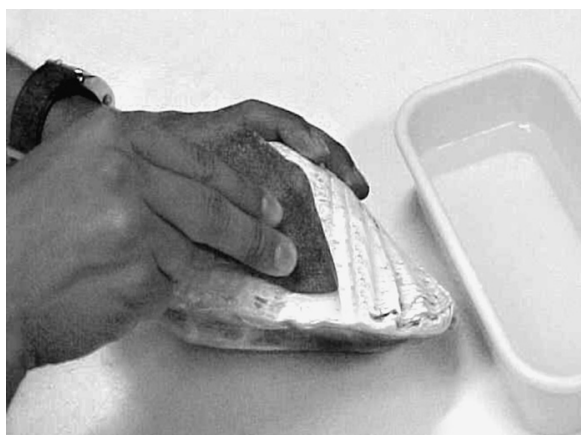

d

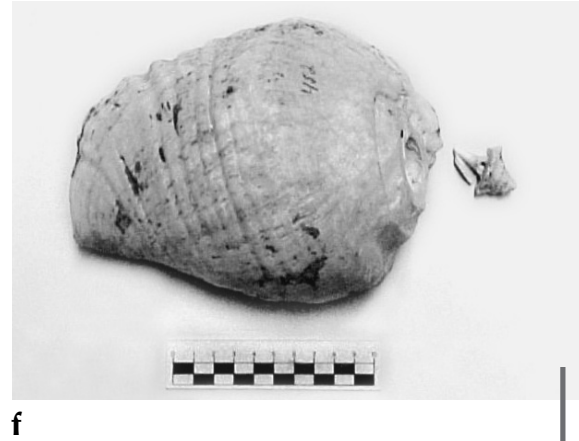

Figura 4 - Ejemplos de procesos de corte y desgaste de conchas con la herramienta arqueológica de pizarra: a) valva de Pinctada mazatlanica con la herramienta; b) proceso de desgaste superficial; c) proceso de corte de charnela; d) desgaste de labio externo de Strombus galeatus; e) proceso de corte de espira de Strombus galeatus, y f) resultado del corte de espira 
En el caso de los bivalvos, ambos procesos fueron realizados en el mismo ejemplar biológico, iniciándose siempre con el desgaste, el cual fue hecho frotando la herramienta sobre la superficie externa de las conchas, con movimientos de arriba hacia abajo y laterales, adicionando agua constantemente; en el caso de la Pinctada mazatlanica esta modificación se llevó a cabo hasta que pudo descubrirse totalmente el endostraco nacarado; en el del Spondylus princeps se hizo solamente hasta remover las espinas y obtener algunas zonas alisadas; en el de la Anadara grandis la superficie fue trabajada hasta eliminar por completo las costillas radiales. En lo referente al Strombus galeatus, se llevó a cabo el corte de la espira, mediante el proceso anteriormente descrito, y posteriormente se desgastó un área próxima al labio externo del mismo caracol. En la tabla 1 se resumen los tiempos que tomó cada uno de los experimentos realizados. Es importante comentar algunos aspectos acerca de las diferencias en los tiempos de trabajo de los experimentos. En el caso de la Pinctada mazatlanica la mayor inversión de trabajo en el desgaste de su superficie, con relación a los demás experimentos, se debe a que para descubrir el endostraco nacarado es necesario eliminar totalmente dos capas de la concha, la externa (periostraco) y la media (meostraco), cosa que no ocurre en ninguna de las otras especies. Con respecto a la Anadara grandis, las 2 horas y media que demoró la supresión de su ornamentación natural se debe a que se experimentó con un ejemplar de pequeñas dimensiones, muy diferente a los grandes especímenes que se encuentran en Rica Playa. En el caso del corte de la charnela en Spondylus princeps, su tiempo de trabajo es mayor debido a que las conchas de esta especie son en general masivas. Finalmente, es importante mencionar que los tiempos de trabajo de los experimentos no pueden equipararse con los que debieron emplear los artesanos prehispánicos, tanto porque puede suponerse que tuvieron una mayor habilidad, así como una mejor calidad y variedad de herramientas.

Tabla 1 - tiempo de trabajo empleado en los experimentos realizados

\begin{tabular}{|c|c|c|}
\hline $\begin{array}{c}\text { Especie } \\
\text { (largo, ancho y alto en } \mathrm{cm})\end{array}$ & $\begin{array}{c}\text { Tiempo de } \\
\text { desgaste }\end{array}$ & Tiempo de corte \\
\hline $\begin{array}{c}\text { Pinctada mazatlanica } \\
(10,7 ; 9,8 ; 1,7)\end{array}$ & 6 horas & $1: 20$ horas \\
\hline $\begin{array}{c}\text { Anadara grandis } \\
(5 ; 7,65 ; 2,35)\end{array}$ & $2: 30$ horas & $3: 45$ horas \\
\hline $\begin{array}{c}\text { Spondylus princeps } \\
(8,1 ; 8,2 ; 2,5)\end{array}$ & $0: 30$ horas & $4: 55$ horas \\
\hline $\begin{array}{c}\text { Strombus galeatus } \\
(18,7 ; 13,6 ; 9,1)\end{array}$ & $3: 15$ horas & $3: 10$ horas \\
\hline
\end{tabular}

Una vez realizadas las experiencias, se tomaron réplicas en polímeros tanto de las modificaciones experimentales como de las arqueológicas. Se obtuvieron un total de 33 muestras, 25 de las cuales corresponden a los cortes y desgastes de las piezas de Rica Playa, y las restantes a los experimentos. La obtención de réplicas en polímeros es una técnica propia de la metalografía, que consiste en presionar un acetato reblandecido con acetona contra la superficie que se desee analizar; en éste se marcan los rasgos superficiales del material con un gran nivel de detalle. Posteriormente dichas muestras se recubren con oro para poder ser vistas en el microscopio electrónico de barrido en el modo de alto vacío. La aplicación de esta técnica no intrusiva tiene varias ventajas en la arqueología, como el poder observar piezas que por su talla nunca podrían ingresar en la cámara de muestreo, o modificaciones que por su forma resultarían muy problemáticas; además evitan los riesgos de los traslados de los materiales al laboratorio, 
así como los trámites burocráticos que ello conlleva, ya que las réplicas pueden obtenerse en los mismos repositorios en que se resguardan los materiales. Las réplicas fueron observadas en un microscopio marca Jeol, modelo JSM-6460LV, que se encuentra en la Subdirección de Laboratorios y Apoyo Académico del Instituto Nacional de Antropología e Historia de México, en modo de alto vacío, con una potencia de $20 \mathrm{KV}$, una apertura de haz de 42 y una distancia de trabajo de $10 \mathrm{~mm}$; se obtuvieron cuatro imágenes digitales de cada muestra, a 100X, 300X, $600 x$ y $1000 x$.

\section{RESULTADOS}

En el caso de las piezas experimentales, los desgastes realizados con la herramienta de pizarra, producen líneas muy finas, del orden de las 0,66 a $1 \mu \mathrm{m}$ de anchura, las cuales corren en sucesión ininterrumpida por toda la superficie, aglomerándose ocasionalmente para formar rasgos de mayores dimensiones. Este mismo patrón puede distinguirse en las paredes de los cortes. Las huellas de trabajo observadas en los materiales arqueológicos coinciden plenamente con los rasgos anteriormente descritos, en todos los casos (figs. 5-12).

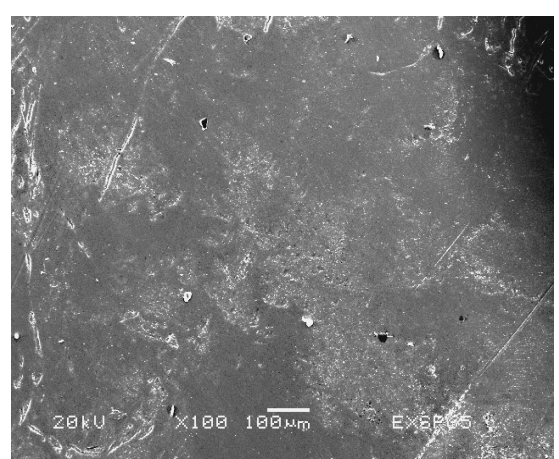

a

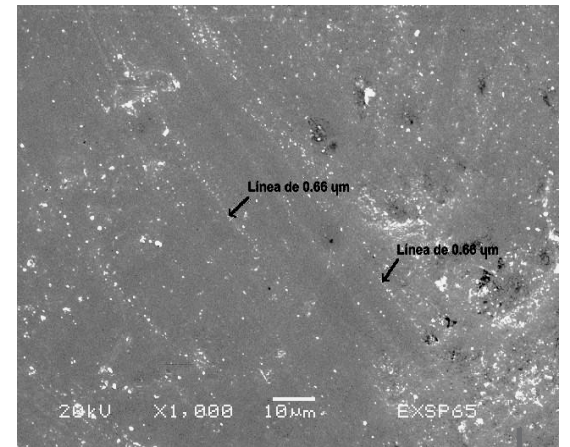

b

Figura 5 - Micrografías a $100 \mathrm{X}$ (a) y $1000 \mathrm{X}$ (b) de las huellas producidas experimentalmente por el desgaste superficial de una valva de Spondylus princeps, con la herramienta de pizarra

Se aprecia una superficie cruzada por finas líneas rectas de aproximadamente $0,66 \mu \mathrm{m}$ de anchura

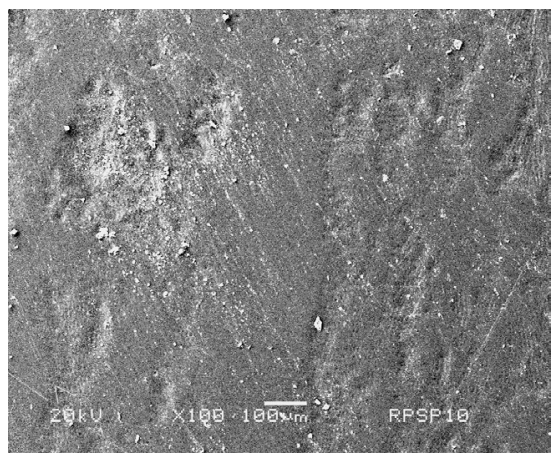

a

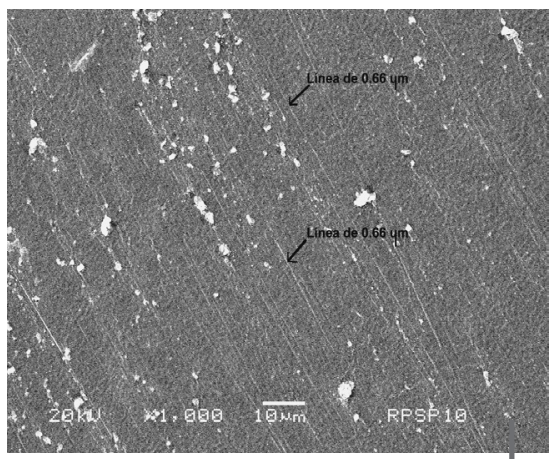

b

Figura 6 - Micrografías a $100 X$ (a) y $1000 X$ (b), de la superficie de un fragmento arqueológico de Spondylus princeps, en el que se aprecia un área cruzada por finas líneas rectas de alrededor de 0,66 $\mu \mathrm{m}$.

Nótese la coincidencia con los rasgos hechos experimentalmente con la herramienta de pizarra en una valva de la misma especie (fig. 5) 
Análisis de las huellas de manufactura del material malacológico de Tumbes, Perú

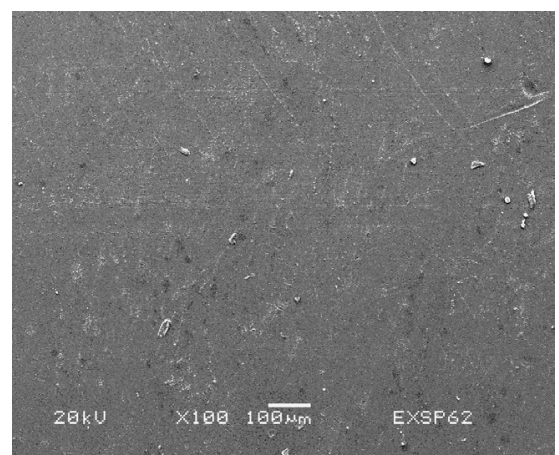

a

Figura 7 - Micrografías a $100 \mathrm{X}$ (a) y $1000 \mathrm{X}$ (b), de un corte hecho experimentalmente con la herramienta de pizarra en una valva de Spondylus princeps.

Se aprecia una superficie cruzada por finas líneas rectas de aproximadamente $0.66 \mu \mathrm{m}$ de ancho, que corren en distintas direcciones

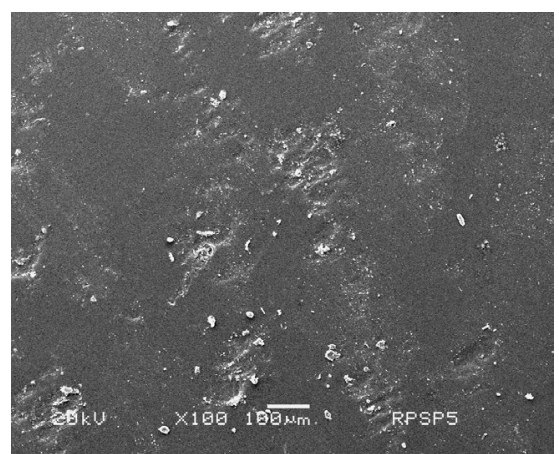

a

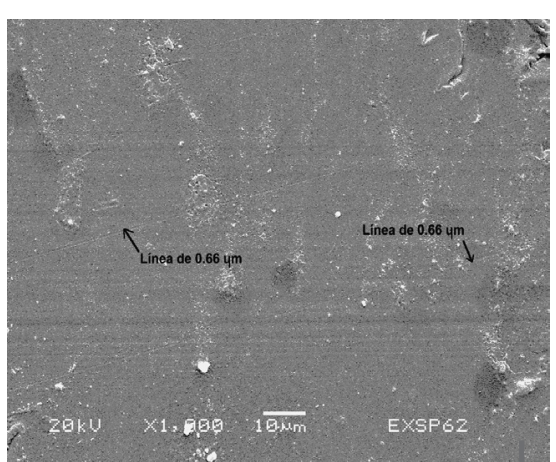

b

Figura 8 - Micrografías a $100 \mathrm{X}$ (a) y $1000 \mathrm{X}$ (b), del corte de un fragmento arqueológico de Spondylus princeps, en el que se aprecia una superficie cruzada por finas líneas rectas de alrededor de 0,66 $\mu \mathrm{m}$. Nótese la coincidencia con los rasgos hechos experimentalmente con la herramienta de pizarra en una valva de la misma especie (fig. 7)

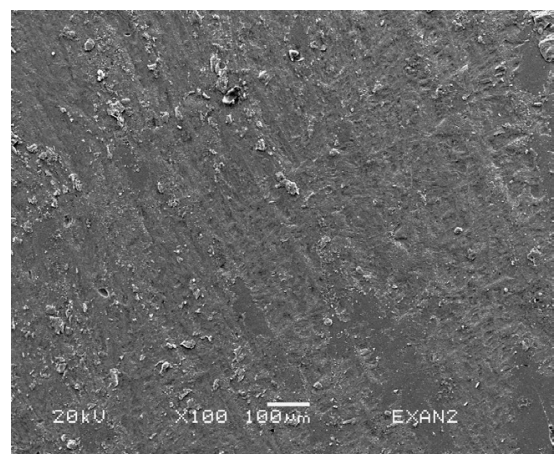

a

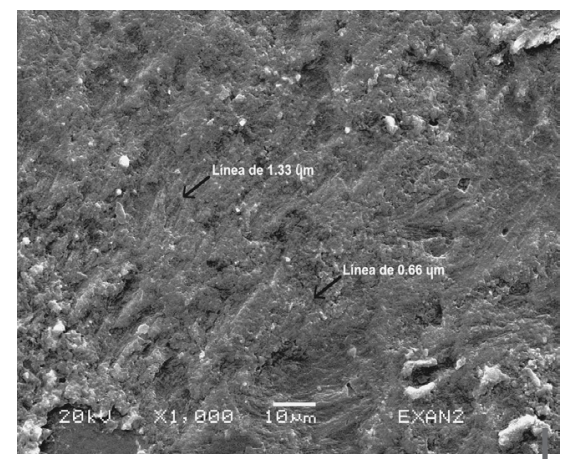

b

Figura 9 - Micrografías a $100 X$ (a) y $1000 X$ (b), de una superficie desgastada experimentalmente con la herramienta de pizarra en una valva de Anadara grandis.

Se aprecia un área cruzada por líneas rectas de entre 0,66 y $1,33 \mu \mathrm{m}$, las cuales se hallan entre los alineamientos cristalinos de la concha 


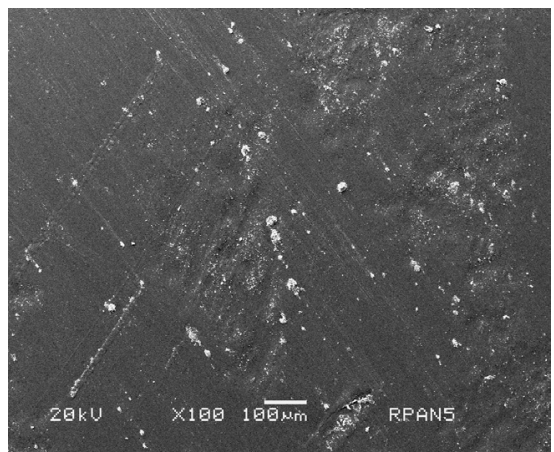

a

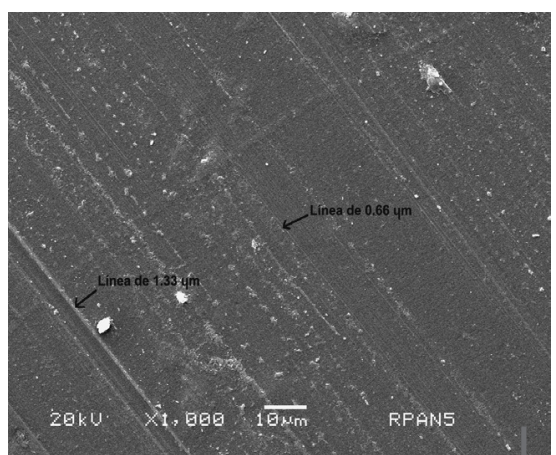

b

Figura 10 - Micrografías a $100 X$ (a) y $1000 X$ (b) de la superficie de un fragmento arqueológico de valva de Anadara grandis, en que se aprecia un área cruzada por líneas rectas de entre 0,66 y 1,33 $\mu \mathrm{m}$ de ancho. Nótese la similitud con la superficie desgastada experimentalmente con pizarra (fig. 9), a pesar de que en este caso la estructura cristalina de la concha no es tan evidente

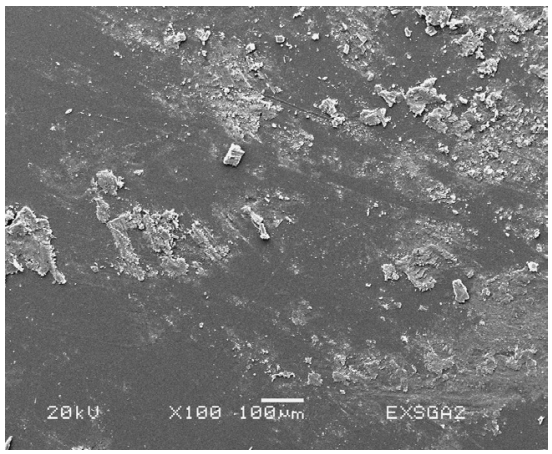

a

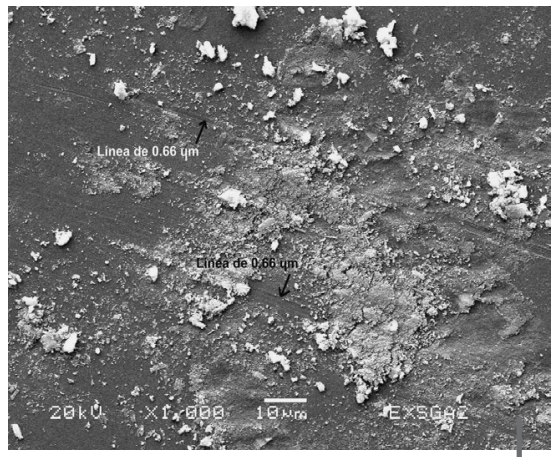

b

Figura 11 - Micrografías a $100 \mathrm{X}$ (a) y $1000 \mathrm{X}$ (b) del corte hecho experimentalmente en un Strombus galeatus, con la herramienta de pizarra.

Se aprecia una superficie cruzada por líneas finas de alrededor de 0,66 $\mu \mathrm{m}$ de anchura

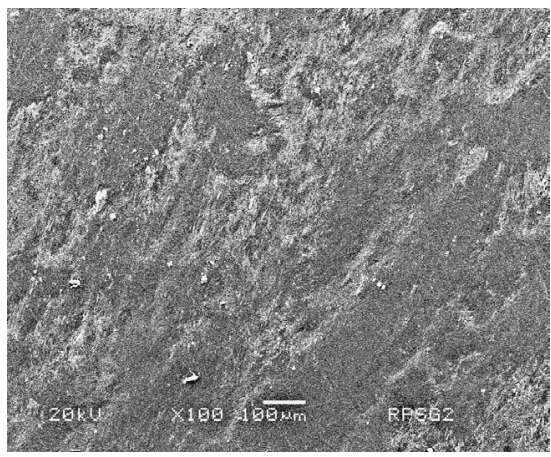

a

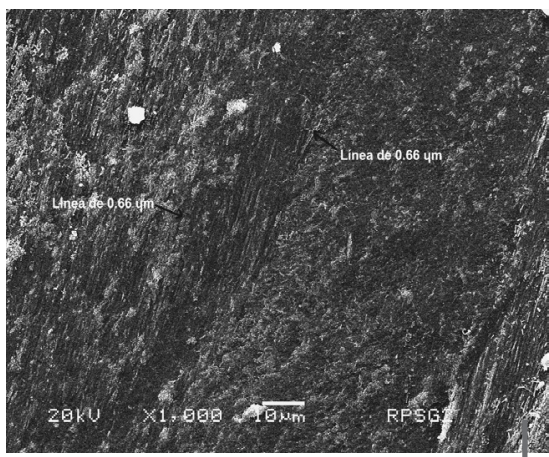

b

Figura 12 - Micrografías a $100 \mathrm{X}$ (a) y $1000 \mathrm{X}$ (b), del corte del fragmento arqueológico de Strombus galeatus, en el que se aprecia una superficie cruzada por finas líneas rectas de alrededor de $0,66 \mu \mathrm{m}$.

Nótese la coincidencia con los rasgos hechos experimentalmente con la herramienta de pizarra en un ejemplar de la misma especie (fig. 11) 
Para hacer más convincente el empleo de herramientas de pizarra para trabajar los materiales de Rica Playa, se presentan ejemplos de huellas de manufactura realizadas en modificaciones similares a las que muestran las piezas del mencionado sitio, existentes en el banco de información del proyecto «Técnicas de manufactura de los objetos de concha del México Prehispánico». Así, por ejemplo, los desgastes con rocas basálticas producen bandas redondeadas e irregulares del orden de las $100 \mu \mathrm{m}$ de anchura (fig. 13a), mientras que la caliza genera rasgos de tres dimensiones: bandas de 30 a $60 \mu \mathrm{m}$ de espesor y líneas rectas de $6 \mu \mathrm{m}$ de ancho (fig. 13b). En cuanto a los cortes, el empleo de lascas afiladas de obsidiana deja una sucesión ininterrumpida de líneas finas de entre 1,3 y $2 \mu \mathrm{m}$ de ancho (fig. 14a), diferentes a las producidas con herramientas similares de pedernal, que dan por resultado bandas de alrededor de 3,3 $\mu \mathrm{m}$ de espesor, que se superponen produciendo rasgos mayores con micros rayados internos (fig. 14b).Como puede apreciarse en las micrografías anteriores, la morfología de los rasgos difiere notablemente de los que presentan las piezas de Rica Playa.

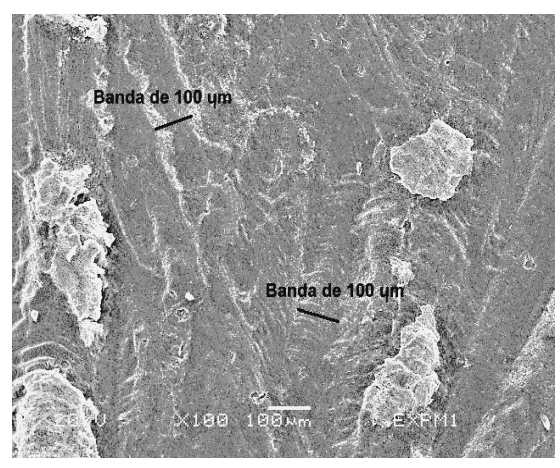

a

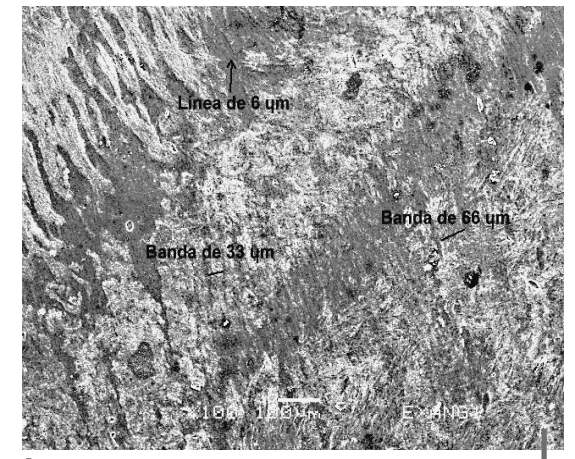

b

Figura 13 - Micrografías a 100X de desgastes superficiales hechos experimentalmente con basalto, en Pinctada mazatlanica (a) y roca caliza, en Anadara grandis (b); en el primer caso se aprecian bandas redondeadas del orden de las $100 \mu \mathrm{m}$ de ancho, mientras que en el segundo se observan tres tipos de rasgos: bandas de aproximadamente 33 y $66 \mu \mathrm{m}$ de espesor, y líneas de $6 \mu \mathrm{m}$ de ancho

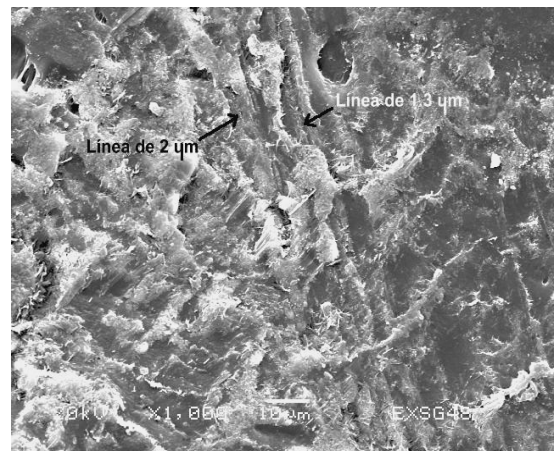

a

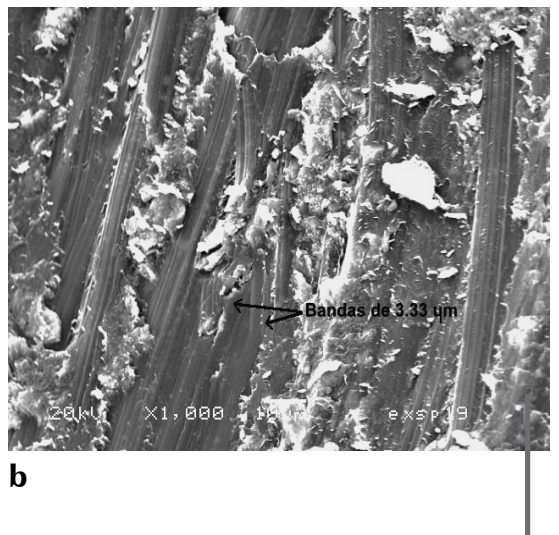

Figura 14 - Micrografías a $\mathbf{1 0 0 0 X}$, de cortes hechos experimentalmente con lascas de obsidiana, en Strombus gigas (a), y con lascas de pedernal, en Spondylus princeps (b); en el primer caso se aprecian líneas finas de entre 1,3 y $2 \mu \mathrm{m}$ de ancho, mientras que en segundo se observan bandas rectas del orden de $3 \mu \mathrm{m}$ de espesor, que se superponen formando rasgos de mayores dimensiones, con micros rayados internos 


\section{CONCLUSIONES}

El análisis de los materiales de Rica Playa ha permitido confirmar una vez más, que la arqueología experimental, junto con la caracterización y comparación de huellas de trabajo, permiten definir con bastante precisión los materiales empleados para la producción de objetos de concha. En este caso en particular, se contó con una ventaja inusual, que fue la de poder trabajar con una de las herramientas arqueológicas con la que se suponía que se llevaba a cabo la elaboración de objetos. Si bien existían ya razones bien fundamentadas para suponer el empleo de utensilios de pizarra en esta industria, el estudio llevado a cabo en esta ocasión permitió confirmar dicha hipótesis. Consideramos que este tipo de corroboraciones son necesarias, ya que en muchas ocasiones la asociación contextual de distintos artefactos, no significa necesariamente su empleo sistémico.

Finalmente, es importante mencionar que el empleo de herramientas de pizarra no ha sido detectado hasta el momento en ninguna de las colecciones mexicanas estudiadas, por lo que podría proponerse como una característica tecnológica andina.

\section{Referencias citadas}

GOSSELAIN, O. P., 1992 - Technology and Style: Potters and Pottery Among Bafia of Camerun. Man, 27: 559-582.

HOCQUENGHEM, A. M., 1993 - Rutas de intercambios del «mullu». Bulletin de I'Institut Français d'Études Andines, 22 (3): 701-719; Lima.

HOCQUENGHEM, A. M., 1994 - Los españoles en los caminos del extremo norte del Perú en 1532. Bulletin de I'Institut Français d'Études Andines, 22 (3): 701-719; Lima.

HOCQUENGHEM, A. M., 1998 - Para Vencer la Muerte. Piura y Tumbes: Raíces en el bosque seco y en la selva alta, horizontes en el Pacífico y en la Amazonía, 445 p.; Lima: CNRS, IFEA, INCAH.

HOCQUENGHEM, A. M., 1999 - En torno al Mullu, manjar predilecto de los poderosos inmortales. In: Spondylus: Ofrenda sagrada y símbolo de paz: 47-102; Lima: Museo Arqueológico Rafael Larco Herrera, Fundación Telefónica del Perú.

HOCQUENGHEM, A. M. \& PEÑA RUIZ, M., 1994 - La talla del material malacológico en Tumbes. Bulletin de I'Institut Français d'Études Andines, 23 (2): 209-229; Lima.

HOLM, O., 2001 - Lanzas Silbadoras y otras contribuciones de Olaf Holm al estudio del pasado del Ecuador (Stothert, K., ed.); Ecuador: Banco Central del ecuador, Museo Antropológico y de Arte Contemporáneo de Guayaquil.

KAUFFMANN DOIG, F., 1987 - Notas arqueológicas sobre la costa extremo norte. Boletín de Lima, 49: 53-57; Lima.

LEMONNIER, P., 1986 - The Study of Material Culture Today. Towards an Anthropology of Technical Systems. Journal of Anthropological Archaeology, (5): 147-186.

LEROI-GOURHAN, A., 1943 - L'homme et la Matière, 367 p.; Paris: Editions Albin Michel.

LEROI-GOURHAN, A., 1945 - Milieu et Techniques, 512 p.; Paris: Editions Albin Michel. 
PEÑA RUIZ, M., 1993 - Taller Malacológico de Cabeza de Vaca. Industria artesanal del Spondylus y otras hijas del mar. Técnica de los Tumbes prehispánicos. In: Primer Congreso Regional de Arqueología y Etnohistoria del Norte Peruano Josefina Ramos de Cox. Piura: 4-19 de junio de 1993. Manuscrito.

PETERSEN, G., 1962 - Las primeras operaciones militares de Francisco Pizarro en el Perú, vol. II: 359-383; Lima. Actas y Trabajos del II Congreso Nacional de Historia del Perú 4-9 de agosto 1958.

PFAFFENBERGER, B., 1988 - Fetishised Objects and Humanizade Nature: Towards an Anthropology of Technology. Man, (23): 236-252.

SCHIFFER, M. B., 1992 - Technological Perspectives on Behavioral Change; Tucson \& Londres: University of Arizona Press.

STARK, M., 1999 - Social Dimensions of Technical Choice in Kalinga Ceramic Tradition. In: Material Meanings (Chilton, E., ed.): 24-43; Salt Lake City: University of Utah Press.

VELÁZQUEZ CASTRO, A., 1999 - Arqueología experimental en materiales conquiológicos; México: Museo del Templo Mayor, Instituto Nacional de Antropología e Historia. Informe final.

VELÁZQUEZ CASTRO, A., 2004 - Técnicas de manufactura de los objetos de concha del Templo Mayor de Tenochtitlan. La producción especializada de los objetos de concha del Templo Mayor de Tenochtitlan. Facultad de Filosofía y Letras, Universidad Nacional Autónoma de México. Tesis de Doctorado en Antropología.

VELÁZQUEZ CASTRO, A., MENDOZA ANAYA, D. \& VALENTÍN MALDONADO, N., 2004 - Los Anáhuatl de concha del Templo Mayor de Tenochtitlan: su valor visto a través de sus técnicas de manufactura. In: La Ciencia de Materiales y su impacto en la Arqueología (Mendoza Anaya, D., Brito Benítez,E. L. \& Arenas Alatorre, J. A., eds.): 129-140; México: Academia Mexicana de Ciencias Materiales.

ZEVALLOS MENÉNDEZ, C., 1995 - Nuestras Raíces Guancavilcas, 435 p.; Guayaquil: Casa de la Cultura, Núcleo del Guayas, con el auspicio de la Muy Ilustre Municipalidad de Guayaquil. 